\title{
REVIEWS
}

Anna Turska-Szybka ${ }^{\text {A-C, E, F, Paulina Anna Pasternok }}{ }^{\mathrm{B}-\mathrm{D}}$, Dorota OlCZaK-KOWALCZYK ${ }^{\text {E, F }}$

\section{Xylitol Content in Dental Care and Food Products Available on the Polish Market and Their Significance in Caries Prevention}

\section{Zawartość ksylitolu w produktach pielęgnacyjnych i spożywczych dostępnych na polskim rynku a profilaktyka choroby próchnicowej}

Department of Pedodontics, Medical University of Warsaw, Warsaw, Poland

A - research concept and design; $\mathbf{B}$ - collection and/or assembly of data; $\mathbf{C}$ - data analysis and interpretation;

$\mathbf{D}$ - writing the article; $\mathbf{E}$ - critical revision of the article; $\mathbf{F}$ - final approval of article

\begin{abstract}
Caries pertains to most of the Polish community and is associated with a high intake of sucrose and monosaccharides. Their non-cariogenic analogue is xylitol - equally sweet as sucrose, but not metabolized by the majority of bacteria existing in the oral cavity.

The aim of this paper is to evaluate the content of xylitol in dental care and food products available on the Polish market and their significance in caries prevention. The products were selected based on the literature, informational leaflets and information from manufacturers. The toothpastes and gels taken into consideration contain up to $25 \%$ xylitol, mouth rinses - $25 \%$, dental care sprays - 35\%, chewing gum - 66-67\% and candies - $96 \%$. A large number of dental care and food products recommended in caries prevention contain xylitol in diverse concentrations. If caries-producing sugars are replaced with non-cariogenic sugar alcohols, the frequency of caries may be diminished. On one hand, xylitol, as an acknowledged anti-carious agent, is included in many caries-prevention programs, in precise concentrations and regularly used, on the other hand - there is a lack of EBM (Evidence-Based Medicine) data indicating the effectiveness of xylitol, apart from those pertaining to fluoride toothpastes with the addition of xylitol. It is emphasized that more detailed trials are necessary to answer the question whether it is xylitol that is relevant in caries prevention or the alteration of habits - lessened sucrose intake or its complete substitution with a sweetener and the act of chewing, which stimulates salivation (Dent. Med. Probl. 2016, 53, 4, 542-550).
\end{abstract}

Key words: xylitol, caries prevention, chewing gum with xylitol, lozenges with xylitol, toothpaste with xylitol.

Słowa kluczowe: ksylitol, profilaktyka próchnicy, guma do żucia z ksylitolem, pastylki do ssania z ksylitolem, pasta do zębów z ksylitolem.

Sugar alcohols (or polyols), natural organic compounds, may be used in order to replace sucrose. There are many sugar alcohols administered in dental care and food products, such as maltitol, lactitol, sorbitol, mannitol, erythritol and xylitol.

Xylitol (E 967) is a five-carbon sugar alcohol of crystalline structure and sweetness similar to sucrose and has the same relative sweetness, which is 1 , and provides less energy compared to sucrose (2.4 kcal vs $4 \mathrm{kcal})$. It occurs naturally in certain fruits, such as plums, raspberries and strawberries, and in birch trees; it is also developed in the digestive reactions of the human body in the amount of $15 \mathrm{~g}$ /day. It can be industrially produced using products rich in xylans, such as corncobs and birch wood. Xylitol obtained in the reduction reaction of xylose in the presence of catalytic converters is used in the food and cosmetic industry as an ingredient of chewing gum, lozenges, sprays, mouth rinses, gels, toothpastes, wipes, candies and 
varnishes for professional use (according to Regulation No. 1333/2008 of the European Parliament and Council of 16 December 2008 on food additives and the directive of the Minister of Health of 22 November 2010 on permitted food additives).

According to American Academy of Pediatric Dentistry (AAPD), xylitol reduces the levels of Streptococcus mutans (SM) both in the plaque and saliva [1]. Xylitol impairs the glycolysis in SM, resulting in its energy cycle being ineffectual and leading to cell death. Furthermore, it has been proved that consuming effective doses of xylitol causes a diminution of the adhesive properties of SM and reduces other virulence factors, for example lessened acid production. In contrast to other sweeteners, it is not a substrate for the majority of bacteria existing in the oral cavity but a passive substitute for cariogenic sugars. It manifests anticarious action principally against Streptococcus mutans (SM) [2]. It inhibits the growth of SM in the presence of glucose, fructose, mannose, sorbitol and lactose, both in saliva and dental plaque, and decreases the amount of dental plaque [2-6]. Xylitol affects the adhesion of SM to the enamel.

It has been found that long-term administration may induce the onset of resistant strains, but of lessened virulence compared to the sensitive strains [7]. The metabolism of glucose and production of lactate may recur to previous levels. In the case of sensitive bacteria, it is absorbed into the cell wall of SM as xylulose-5-phosphate (X5P) and inhibits the enzymes of sugar metabolism, reducing the acid-forming properties of the environment of the oral cavity.

Administration of xylitol delays the mother-to-child transmission of SM $[2,5]$. According to the AAPD, it is recommended to use 3-8 $\mathrm{g}$ of xylitol per day only in syrup form in children below 4 years of age, above that age $-3-8$ g per day in chewing gum, lozenges, candies and snacks, in both cases divided into at least 2 doses [1]. The findings indicate that applying xylitol syrup in the amount of $8 \mathrm{~g}$ daily compared to $2.67 \mathrm{~g}$ decreases the incidence of caries by $58 \%$ in children [8]. It has been proved that administering about $10 \mathrm{~g}$ of xylitol per day provides the greatest benefit [1]. It has been established that using a large dose of xylitol (11.6 g/day) in chewing gum for 6 months in children at high risk of caries is enough to reduce the increment of decayed surfaces in first permanent molars for 2 years [9]. Studies have also proven that applying $5 \mathrm{~g}$ of xylitol per day divided into 5 doses impacts the depletion of root caries in adults by $40 \%$ (compared to an untreated group) [10]. This may result from the ceiling effect - using more than about $10 \mathrm{~g}$ a day has no justification in the significant reduction of the quantity of SM in saliva. According to Milgrom et al. [11], xylitol in the amount of $6.44 \mathrm{~g}$ per day and $10.32 \mathrm{~g}$ per day may reduce the quantity of SM in dental plaque over 5 weeks from the initiation of application, and in resting saliva - over 6 months. The authors estimate the ceiling effect for xylitol between $6.44 \mathrm{~g}$ and $10.32 \mathrm{~g}$ per day. Another study has shown that xylitol intake of $10.32 \mathrm{~g}$ daily in chewing gum yields a significant reduction of SM in resting saliva and dental plaque and that an inverse proportion occurs between the frequency of chewing xylitol gum and bacteria count [12]. Oscarson et al. [13] have acknowledged that applying $0.48 \mathrm{~g}$ per day and, after 6 months, $0.96 \mathrm{~g}$ per day in children over 2 years old has no significant preventive anticarious effect.

For xylitol, as with other polyols, an acceptable daily intake has not been determined. However, it is not absorbed in the small intestine and progresses to the colon where it ferments. Excessive intake (more than 25-40 g per day) may induce loose stool or diarrhea. Other adverse reactions are oral ulcers, cramps, meteorisms and constipation [8]. Symptoms from the gastrointestinal tract are usually observed at the beginning of using the sweetener, and they may be minimized by gradual increase of the daily dose.

Xylitol remains a questionable means in caries prevention. In the USA, xylitol is included in many public and private dental prophylactic programs based on recommendations according to guidelines, both for adults and children (AAPD 2014/15; ADA 2011) [1, 14]. However, in many European countries its application is not always embodied in guidelines concerning caries prevention [8]. It is denoted that more precise studies are necessary to clarify whether it is xylitol that is significant in caries prevention or the alteration of habits (lessened sucrose intake or its complete substitution with a sweetener) and the act of chewing stimulating the salivary flow [15]. Due to its pleasant taste, xylitol increases the flow of saliva, providing calcium, phosphate and fluoride ions for enamel remineralization and acting as a buffer.

The passive effects of using xylitol as a sugar equivalent should not be neglected. Fontana and González-Cabezas [16] have indicated a lower SM possession in mothers after application of chewing gum containing xylitol in comparison to chewing gum with sorbitol and without the application of chewing gum, yet the differences were not statistically significant. Also, the sort of xylitol-containing product and the manner of its consumption (chewing gum, lozenges, syrup, etc.) was significant [17]. The impact of additives to products containing xylitol on the development of oral microbiota is also worth noting. Regrettably, the results 
of the majority of the available research are evaluated to be of low and very low quality and accordingly, it is difficult to assess whether xylitol-containing products are an efficient means in caries prevention in children, youth and adults [8].

\section{Aim of the Study}

The aim of this paper is to evaluate the content of xylitol in dental care and food products available on the Polish market and their significance in caries prevention.

\section{Materials and Methods}

Both dental care and food products containing xylitol were selected based on the literature, informational leaflets and information from manufacturers. In the case of some products available on the Polish market, it was ultimately impossible to ascertain the exact percent composition of xylitol and such products were not included in this paper. The paper does not encompass all xylitol-containing products available on the Polish market.

In the tables, optimal dosages (according to the AAPD) of 3-8 g per day were taken into consideration; only in syrup form for children below 4 years old, and for children above 4 years old, all appropriate products, such as chewing gum, candies, snacks, toothpaste, mouth rinses and other dental care products [1]. However, it is worth emphasizing that, according to the thesis and results of Milgrom et al. [11], "a dose of $3.44 \mathrm{~g} /$ day or less is not likely to show changes in mutans streptococci level".

In the case of toothpastes, 1 serving is stated to be $1-2 \mathrm{~cm}$ of the product, or a pea-sized portion, for Nenedent-baby, Dentinox ${ }^{\circledR}$ (Miralex) toothpaste. It was assumed that a portion of toothpaste or gel $(1-2 \mathrm{~cm})$ is approximately $0.5 \mathrm{~g}$; the size of a pea was estimated at about $0.25 \mathrm{~g}$. On this basis, achieving the optimal daily dosage of xylitol by administering only toothpaste is impossible (this would necessitate applying more than 1 tube of toothpaste daily), therefore the amount provided while brushing twice per day has been calculated.

If the mass of 1 piece of chewing gum was not specified by its manufacturer, it was presupposed that it amounts to $1.5 \mathrm{~g}$ (the most common weight of 1 piece of chewing gum). Regarding other situations, the information proffered by the manufacturer is included in the table. In the matter of lozenges, it was presumed that 1 piece weighs $1 \mathrm{~g}$ (the most common weight); if the manufacturer provided different data, it is included in the table.

\section{Results}

\section{Dental Care Products}

Toothpastes and gels contain between 10 and $25 \%$ of xylitol, as presented in Table 1.

Table 2 shows that mouth rinses contain up to $25 \%$ of xylitol, sprays $-35 \%$.

\section{Food Products}

Chewing gum contains $66-67 \%$ of xylitol, as presented in Table 3.

Candies and lozenges had the highest concentrations of xylitol, between $83 \%$ and $96 \%$, as presented in Table 4.

There are many alternatives to popular snacks available, containing xylitol (Table 5).

\section{Discussion}

Dental care products intended for oral hygiene, including toothpastes and gels, contain up to $25 \%$ xylitol, mouth rinses - up to $25 \%$, sprays - up to $35 \%$, whereas chewing gum contains considerably more $-66-67 \%$. The product of the most substantial percent composition of xylitol is a candy KsyliCuksy $^{\circledR}(96 \%)$. Among snacks, both products containing large amounts of xylitol and products with lower percentages of xylitol were retrieved.

Ingestion of the recommended daily dose of xylitol using only one type of product is not always manageable. Due to this fact, in daily life it is worth considering to combie different kinds of xylitol-containing products in order to provide the optimal amount of the sweetener, based on the products of its highest concentration, using the others to complement the provision. Regarding children at high risk of developing early childhood caries (ECC), applying a toothpaste containing fluoride and xylitol compared to applying a toothpaste consisting of fluoride and sorbitol does not demonstrate a significant efficacy in reducing ECC [18]. However, in vitro studies suggest that combining fluoride and xylitol in toothpastes aids enamel remineralization [19]. Even so, in a metaanalysis by the ADA, it is stated that there is not enough evidence to acknowledge xylitol-containing dentifrices as preventing caries [14].

Studies on the efficacy of mouth rinses with xylitol have proven that administering a mouthwash containing $20 \%$ of xylitol 5 times per day for 1 minute over 4 weeks significantly lowers the SM count [20]. Preparing a mouth rinse at home requires dissolving $1 \mathrm{~g}$ of xylitol in $10 \mathrm{~mL}$ of water [21]. Nevertheless, it is believed that xylitol applied in 
Table 1. Toothpastes and gels

\begin{tabular}{|c|c|c|c|c|c|c|c|}
\hline & $\begin{array}{l}\text { Product name } \\
\text { (manufacturer) }\end{array}$ & $\begin{array}{l}\text { Xylitol } \\
\text { content } \\
(\%)\end{array}$ & $\begin{array}{l}\text { Directions } \\
\text { from } \\
\text { manufacturer }\end{array}$ & Additives & $\begin{array}{l}\text { Amount } \\
\text { of xylitol in } \\
1 \text { serving }\end{array}$ & $\begin{array}{l}\text { Amount of xylitol } \\
\text { provided while bru- } \\
\text { shing twice a day }\end{array}$ & $\begin{array}{l}\text { Source of } \\
\text { information }\end{array}$ \\
\hline 1. & $\begin{array}{l}\text { CTx3 Gel, CariFree }{ }^{\circledR} \text {, } \\
\text { (Oral Bio Tech USA) }\end{array}$ & 25 & $\begin{array}{l}\text { use twice daily, } \\
\text { no age restric- } \\
\text { tion }\end{array}$ & - & $0.125 \mathrm{~g}$ & $0.25 \mathrm{~g}$ & $\begin{array}{l}\text { manufacturer's } \\
\text { website }\end{array}$ \\
\hline 2. & $\begin{array}{l}\text { Ctx4 Gel, CariFree } \\
\text { (Oral Bio Tech USA) }\end{array}$ & 25 & $\begin{array}{l}\text { over } 6 \text { y. o., use } \\
\text { twice daily }\end{array}$ & $\begin{array}{l}\text { sodium fluoride } \\
(1.1 \%)\end{array}$ & $0.125 \mathrm{~g}$ & $0.25 \mathrm{~g}$ & $\begin{array}{l}\text { manufacturer's } \\
\text { website }\end{array}$ \\
\hline 3. & $\begin{array}{l}\text { All In ONE Win- } \\
\text { tergreen toothpaste, } \\
\text { Yotuel }^{\circledR} \\
\text { (Biocosmetics) }^{\text {Bis }}\end{array}$ & 11 & $\begin{array}{l}\text { use } 3 \text { times } \\
\text { a day, brush } \\
\text { for } 3 \text { minutes }\end{array}$ & $\begin{array}{l}\text { sodium fluoride } \\
(0.32 \%) \text {, carba- } \\
\text { mide peroxide } \\
(0.1 \%)\end{array}$ & $0.055 \mathrm{~g}$ & $0.11 \mathrm{~g}$ & $\begin{array}{l}\text { informational } \\
\text { leaflet }\end{array}$ \\
\hline 4. & $\begin{array}{l}\text { Dental Fluoride Free } \\
\text { Xylitol Toothpaste, } \\
\text { Epic }^{\circledR} \text { (Santini) }\end{array}$ & 25 & $\begin{array}{l}\text { over } 2 \text { y. o., } \\
\text { use minimum } \\
\text { twice a day }\end{array}$ & $\begin{array}{l}\text { stevia, zinc pyr- } \\
\text { rolidiane car- } \\
\text { boxylate }\end{array}$ & $0.125 \mathrm{~g}$ & $0.25 \mathrm{~g}$ & $\begin{array}{l}\text { informational } \\
\text { leaflet }\end{array}$ \\
\hline 5. & $\begin{array}{l}\text { Fluoride-free xylitol } \\
\text { toothpaste, Spry } \\
\text { (Xlear) }\end{array}$ & 25 & $\begin{array}{l}\text { over } 2 \text { y. o., } \\
\text { use minimum } \\
\text { twice a day }\end{array}$ & $\begin{array}{l}\text { aloe, calcium } \\
\text { lactate, parsley } \\
\text { seed oil, stevia }\end{array}$ & $0.125 \mathrm{~g}$ & $0.25 \mathrm{~g}$ & $\begin{array}{l}\text { informational } \\
\text { leaflet }\end{array}$ \\
\hline 6. & $\begin{array}{l}\text { Blooming Sakura, } \\
\text { Refreshing tooth- } \\
\text { paste, }\left(\text { ROCS }^{\circledR}\right)\end{array}$ & 10 & $\begin{array}{l}\text { for everyday } \\
\text { use, over } 4 \text { y. o. }\end{array}$ & $\begin{array}{l}\text { bromelain, } \\
\text { cherry blossom } \\
\text { extract, magne- } \\
\text { sium chloride, } \\
\text { sorbitol, eugenol }\end{array}$ & $0.05 \mathrm{~g}$ & $0.1 \mathrm{~g}$ & $\begin{array}{l}\text { informational } \\
\text { leaflet }\end{array}$ \\
\hline 7. & $\begin{array}{l}\text { Herba Cura }^{\circledR} \\
3 \text { in } 1 \text { Gel (Akuna) }\end{array}$ & 20 & $\begin{array}{l}\text { for everyday } \\
\text { use as tooth- } \\
\text { paste, gel for } \\
\text { gum massage } \\
\text { and, combined } \\
\text { with water, } \\
\text { mouthrinse }\end{array}$ & $\begin{array}{l}\text { thyme, mint, } \\
\text { licorice, nigella } \\
\text { and sage extracts }\end{array}$ & $0.1 \mathrm{~g}$ & $0.2 \mathrm{~g}$ & $\begin{array}{l}\text { informational } \\
\text { leaflet }\end{array}$ \\
\hline 8. & $\begin{array}{l}\text { Nenedent - baby } \\
\text { toothpaste, } \\
\text { Dentinox }{ }^{\circledR} \text { (Miralex) }\end{array}$ & 13 & - & - & $\begin{array}{l}\text { pea-sized } \\
\text { serving } \\
0.0325 \mathrm{~g}\end{array}$ & $0.065 \mathrm{~g}$ & $\begin{array}{l}\text { informational } \\
\text { leaflet }\end{array}$ \\
\hline
\end{tabular}

Table 2. Other dental care products

\begin{tabular}{|c|c|c|c|c|c|}
\hline & $\begin{array}{l}\text { Product name } \\
\text { (manufacturer) }\end{array}$ & $\begin{array}{l}\text { Xylitol } \\
\text { content } \\
(\%)\end{array}$ & Directions from manufacturer & Additives & $\begin{array}{l}\text { Source of } \\
\text { information }\end{array}$ \\
\hline 1. & $\begin{array}{l}\text { Ctx2 spray, } \\
\text { CariFree }{ }^{\circledR} \\
\text { (Oral Bio Tech USA) }\end{array}$ & 35 & $\begin{array}{l}\text { use } 2-3 \text { sprays in mouth as often as } \\
\text { needed, recommended after meals and } \\
\text { before bedtime, no age restrictions }\end{array}$ & - & $\begin{array}{l}\text { manufacturer's } \\
\text { website }\end{array}$ \\
\hline 2. & $\begin{array}{l}\text { Ctx2 wipes, } \\
\text { CariFree }{ }^{\circledR} \\
\text { (Oral Bio Tech USA) }\end{array}$ & $\mathrm{n} / \mathrm{a}$ & $\begin{array}{l}\text { wrap around a finger and gently wipe } \\
\text { teeth and gums, no age restrictions }\end{array}$ & - & $\begin{array}{l}\text { manufacturer's } \\
\text { website }\end{array}$ \\
\hline 3. & $\begin{array}{l}\text { Ctx3 rinse, } \\
\text { CariFree }{ }^{\circledR} \\
\text { (Oral Bio Tech USA) }\end{array}$ & 25 & $\begin{array}{l}10 \mathrm{~mL} \text { two times daily after brushing } \\
\text { and flossing, do not eat, drink, rinse } \\
\text { for } 30 \mathrm{~min} \text { after use }\end{array}$ & sodium fluoride (0.05\%) & $\begin{array}{l}\text { manufacturer's } \\
\text { website }\end{array}$ \\
\hline 4. & $\begin{array}{l}\text { Xylitol-coated dental } \\
\text { floss, Spry }{ }^{\circledR} \text { (Xlear) }\end{array}$ & $\begin{array}{l}\text { Coating } \\
-100\end{array}$ & for everyday use & - & $\begin{array}{l}\text { informational } \\
\text { leaflet }\end{array}$ \\
\hline 5. & $\begin{array}{l}\text { Spearmint Xylitol } \\
\text { Mouthwash, Epic }{ }^{\circledR} \\
\text { (Santini) }\end{array}$ & 25 & use after brushing & zinc pyrrolidiane carboxylate & $\begin{array}{l}\text { informational } \\
\text { leaflet }\end{array}$ \\
\hline 6. & $\begin{array}{l}\text { Xylitol oral rinse, } \\
\text { Spry }^{\circledR} \text { (Xlear) }\end{array}$ & 25 & rinse twice daily for minimum $30 \mathrm{~s}$ & $\begin{array}{l}\text { marigold, camomile, echina- } \\
\text { cea, thyme, european olive } \\
\text { leaves extracts, aloe }\end{array}$ & $\begin{array}{l}\text { informational } \\
\text { leaflet }\end{array}$ \\
\hline
\end{tabular}

n/a - no answer. 
Table 3. Chewing gums

\begin{tabular}{|c|c|c|c|c|c|c|c|c|}
\hline & $\begin{array}{l}\text { Product name } \\
\text { (manufacturer) }\end{array}$ & $\begin{array}{l}\text { Xylitol } \\
\text { content } \\
(\%)\end{array}$ & $\begin{array}{l}\text { Directions from } \\
\text { manufacturer }\end{array}$ & Additives & $\begin{array}{l}\text { Amount } \\
\text { of xylitol in } \\
1 \text { serving } \\
(1 \text { piece })\end{array}$ & $\begin{array}{l}\text { Number } \\
\text { of servings } \\
\text { to achieve } \\
3 \mathrm{~g} \text { per day }\end{array}$ & $\begin{array}{l}\text { Number of } \\
\text { servings to } \\
\text { achieve } \\
8 \text { g per day }\end{array}$ & $\begin{array}{l}\text { Source of in- } \\
\text { formation }\end{array}$ \\
\hline 1. & $\begin{array}{l}\text { Ctx2 chewing } \\
\text { gum, CariFree }{ }^{\circledR} \\
\text { (Oral Bio Tech } \\
\text { USA) }\end{array}$ & 66 & $\begin{array}{l}2 \text { pieces, } \\
3-5 \text { times daily; } \\
\text { recommended af- } \\
\text { ter meals or when } \\
\text { dry mouth or bad } \\
\text { breath occur }\end{array}$ & - & $0.99 \mathrm{~g}$ & 3 & 8 & $\begin{array}{l}\text { manufacturer's } \\
\text { website }\end{array}$ \\
\hline 2. & $\begin{array}{l}\text { Xylimax }{ }^{\circledR} \text { chewing } \\
\text { gum Trio (Fazer) }\end{array}$ & 67 & - & $\begin{array}{l}\text { titanium } \\
\text { dioxide }\end{array}$ & $\begin{array}{l}1 \text { piece } \\
-1.51 \mathrm{~g} \\
1.01 \mathrm{~g} \\
\end{array}$ & 3 & 8 & $\begin{array}{l}\text { informational } \\
\text { leaflet }\end{array}$ \\
\hline 3. & $\begin{array}{l}\text { Peppermint xylitol } \\
\text { chewing gum, } \\
\text { Spry }^{\circledR} \text { (Xlear) }\end{array}$ & 67 & $\begin{array}{l}\text { for everyday use } \\
\text { after meals }\end{array}$ & $\begin{array}{l}\text { pepper- } \\
\text { mint oil }\end{array}$ & $\begin{array}{l}1 \text { piece } \\
-1.08 \mathrm{~g} \\
0.724 \mathrm{~g}\end{array}$ & 4 & 11 & $\begin{array}{l}\text { informational } \\
\text { leaflet }\end{array}$ \\
\hline 4. & $\begin{array}{l}\text { Xylitol, spearmint } \\
\text { xylitol chewing } \\
\text { gum, Miradent }{ }^{\circledR} \\
\text { (Hager \& Werken) }\end{array}$ & 67 & $\begin{array}{l}\text { use at least } \\
3 \text { times daily, af- } \\
\text { ter meals }\end{array}$ & - & $\begin{array}{l}1 \text { piece } \\
-1.4 \mathrm{~g} \\
0.938 \mathrm{~g}\end{array}$ & 4 & 8.5 & $\begin{array}{l}\text { information } \\
\text { from manufac- } \\
\text { turer }\end{array}$ \\
\hline
\end{tabular}

Table 4. Candies, lozenges

\begin{tabular}{|c|c|c|c|c|c|c|c|c|}
\hline & $\begin{array}{l}\text { Product name } \\
\text { (manufacturer) }\end{array}$ & $\begin{array}{l}\text { Xylitol } \\
\text { content } \\
(\%)\end{array}$ & $\begin{array}{l}\text { Directions } \\
\text { from } \\
\text { manufacturer }\end{array}$ & Additives & $\begin{array}{l}\text { Amount of } \\
\text { xylitol in } \\
1 \text { serving } \\
(1 \text { piece })\end{array}$ & $\begin{array}{l}\text { Number } \\
\text { of servings } \\
\text { to achieve } \\
3 \mathrm{~g} \text { per day }\end{array}$ & $\begin{array}{l}\text { Number } \\
\text { of servings } \\
\text { to achieve } \\
8 \mathrm{~g} \text { per day }\end{array}$ & $\begin{array}{l}\text { Source of } \\
\text { information }\end{array}$ \\
\hline 1. & $\begin{array}{l}\text { Ksyli-Cuksy }^{\circledR} \\
\text { - orange-flavored } \\
\text { candies } \\
\text { (Pięć Przemian) }\end{array}$ & 96 & - & - & $0.96 \mathrm{~g}$ & 3 & 8 & $\begin{array}{l}\text { informational } \\
\text { leaflet }\end{array}$ \\
\hline 2. & $\begin{array}{l}\text { Ksyli-Cuksy }{ }^{\circledR}-\text { ca- } \\
\text { rob candies (Pięć } \\
\text { Przemian) }\end{array}$ & 92 & - & $\begin{array}{l}\text { carob (5\%), } \\
\text { inulin (3\%) }\end{array}$ & $0.92 \mathrm{~g}$ & 3 & 9 & $\begin{array}{l}\text { informational } \\
\text { leaflet }\end{array}$ \\
\hline 3. & $\begin{array}{l}\text { Gems, berry } \\
\text { flavored, Spry } \\
\text { (Xlear) }\end{array}$ & 83 & $\begin{array}{l}1-2 \text { pieces } \\
\text { after every } \\
\text { meal }\end{array}$ & - & $\begin{array}{l}1 \text { piece } \\
-0.6 \mathrm{~g} \\
0.498 \mathrm{~g}\end{array}$ & 6 & 16 & $\begin{array}{l}\text { informational } \\
\text { leaflet }\end{array}$ \\
\hline 4. & $\begin{array}{l}\text { Tingz, xylitol } \\
\text { sweets, strawberry } \\
\text { and vanilla (Pep- } \\
\text { persmith }{ }^{\circledR} \text { ) }\end{array}$ & 93 & - & - & $\begin{array}{l}1 \text { piece } \\
-0.6 \mathrm{~g} \\
0.558 \mathrm{~g}\end{array}$ & 5 & 14 & $\begin{array}{l}\text { informational } \\
\text { leaflet }\end{array}$ \\
\hline
\end{tabular}

a mouthwash may not have a significant preventive efficacy due to the short time of exposure to the substance in the oral cavity. Experimental application of $4.3 \mathrm{~g}$ of xylitol in chewing gum three times daily or $4.4 \mathrm{~g}$ in a mouth rinse $(2.2 \mathrm{~g}$ of xylitol in $20 \mathrm{~mL}$ of water twice a day) over 3 months resulted in an insignificant depletion in the count of SM in stimulated saliva [22]. Subramaniam et al. [23] suggest combining sodium fluoride, xylitol and triclosan into a mouth rinse for routine use in children, for the sake of decreasing the amount of SM after 21 days of its application. Decker et al. [24] have noted synergy in the anticarious action of chlorhexidine and xylitol and recommend harnessing such a combination in prophylactic products.
In order to maintain valid oral hygiene in newborns and infants, it is worth considering to use xylitol wipes or sprays instead of a traditional toothbrush with a small amount of toothpaste; such simplification might aid in maintaining oral hygiene in the youngest children. In studies conducted by Zhan et al. [25], daily cleaning with a xylitol wipe over 1 year lead to reducing caries in children by $14 \%$ compared to using non-xylitol wipes [8]. Localized treatment with xylitol after brushing teeth as an addition to daily hygienic habits in children over 6 months brings a reduction in the amount of SM and, at the age of 7, lessened caries frequency [26]. Oral administration of a xylitol syrup (daily dose: 8 g) 2-3 times a day over 1 year results in 
Table 5. Selected food products

\begin{tabular}{|c|c|c|c|c|c|c|c|c|}
\hline & $\begin{array}{l}\text { Product name } \\
\text { (manufacturer) }\end{array}$ & $\begin{array}{l}\text { Xylitol } \\
\text { content } \\
(\%)\end{array}$ & $\begin{array}{l}\text { Directions } \\
\text { from } \\
\text { manufacturer }\end{array}$ & Additives & $\begin{array}{l}\text { Amount } \\
\text { of xylitol in } \\
1 \text { serving }\end{array}$ & $\begin{array}{l}\text { Number } \\
\text { of servings } \\
\text { to achieve } \\
3 \text { g per day }\end{array}$ & $\begin{array}{l}\text { Number } \\
\text { of servings } \\
\text { to achieve } \\
8 \text { g per day }\end{array}$ & $\begin{array}{l}\text { Source } \\
\text { of information }\end{array}$ \\
\hline 1. & $\begin{array}{l}\text { Ctx1 lollies' } \text { Carifree }^{\circledR} \\
\text { (Oral Bio Tech USA) }\end{array}$ & $\mathrm{n} / \mathrm{a}$ & $\begin{array}{l}2 \text { times daily, } \\
\text { recommended } \\
\text { after meals }\end{array}$ & - & $\begin{array}{l}1 \text { piece } \\
-1 \mathrm{~g}\end{array}$ & 3 & & $\begin{array}{l}\text { manufacturer's } \\
\text { website }\end{array}$ \\
\hline 2. & $\begin{array}{l}\text { Makao }^{\circledR} \text { hot choco- } \\
\text { late with xylitol } \\
\text { (Simpatiko) }\end{array}$ & 58 & $\begin{array}{l}\text { combine } 2 \text { te- } \\
\text { aspoons with } \\
\text { milk }\end{array}$ & $\begin{array}{l}35 \% \text { cocoa, } \\
\text { cinnamon, } \\
\text { cardamon }\end{array}$ & $\begin{array}{l}2 \text { teaspoons } \\
-10 \mathrm{~g} \\
5.8 \mathrm{~g}\end{array}$ & 0.5 & 1.5 & $\begin{array}{l}\text { informational } \\
\text { leaflet }\end{array}$ \\
\hline 3. & $\begin{array}{l}\text { Xylitol-sweetened } \\
\text { plum jam } \\
\left(\text { Pięć Przemian }^{\circledR}\right)\end{array}$ & 6.5 & - & cardamon & $\begin{array}{l}1 \text { teaspoon } \\
-12 \mathrm{~g} \\
0.78 \mathrm{~g}\end{array}$ & 4 & 10 & $\begin{array}{l}\text { informational } \\
\text { leaflet }\end{array}$ \\
\hline 4. & $\begin{array}{l}\text { Banana Dairy Free } \\
\text { Hot Chocolate Flakes } \\
\left(\text { Plamil }^{\mathbb{}}\right)\end{array}$ & 20 & $\begin{array}{l}\text { combine } 6-8 \\
\text { teaspoons } \\
\text { with milk }\end{array}$ & $47 \%$ cocoa & $\begin{array}{l}6 \text { teaspoons } \\
-60 \mathrm{~g} \\
12 \mathrm{~g}\end{array}$ & 0.25 & 0.67 & $\begin{array}{l}\text { informational } \\
\text { leaflet }\end{array}$ \\
\hline 5. & $\begin{array}{l}\text { Cykoriada }{ }^{\circledR} \text { instant } \\
\text { coffee (Simpatiko) }\end{array}$ & 50 & $\begin{array}{l}\text { combine } 2 \text { te- } \\
\text { aspoons with } \\
\text { water }\end{array}$ & $\begin{array}{l}32 \% \text { instant } \\
\text { chickory, } \\
13 \% \text { cocoa, } \\
\text { cinnamon, } \\
\text { cardamon }\end{array}$ & $\begin{array}{l}2 \text { teaspoons } \\
-6 \mathrm{~g} \\
3 \mathrm{~g}\end{array}$ & 1 & 2.67 & $\begin{array}{l}\text { informational } \\
\text { leaflet }\end{array}$ \\
\hline 6. & $\begin{array}{l}\text { Roasted almonds } \\
\text { with xylitol and chilli } \\
\left(\text { Pięć Przemian }{ }^{\circledR}\right)\end{array}$ & 5 & - & coconut oil & $\begin{array}{l}1 \text { almond } \\
-1.5 \mathrm{~g} \\
0.075 \mathrm{~g}\end{array}$ & 40 & 106.67 & $\begin{array}{l}\text { informational } \\
\text { leaflet }\end{array}$ \\
\hline 7. & $\begin{array}{l}\text { Dark chocolate with } \\
\text { xylitol } 75 \%\left(\text { Xucker }^{\circledR}\right)\end{array}$ & 23 & - & $75 \%$ сосоа & $\begin{array}{l}1 \text { part }-6 \mathrm{~g} \\
1.38 \mathrm{~g}\end{array}$ & 2 & 6 & $\begin{array}{l}\text { informational } \\
\text { leaflet }\end{array}$ \\
\hline
\end{tabular}

$\mathrm{n} / \mathrm{a}$ - no answer.

a diminution of ECC by $58 \%$ compared to a syrup of lessened xylitol content $[8,27]$. However, the ADA has concluded there is insufficient evidence to acknowledge xylitol syrup as a means of preventing caries in children under 2 years old [14].

The efficacy of using a xylitol-coated dental floss has not yet been assessed, though flossing is the recommended method of removing dental plaque from interproximal spaces.

In order to achieve a preventive effect, a consistent concentration of xylitol in saliva is essential. This may be obtained by frequent application of chewing gum or lozenges, i.e. dissolving or chewed products intended for prolonged holding in the oral cavity [28]. Even so, no significant difference in xylitol concentration in saliva is noted during the first $30 \mathrm{~min}$ of the application between using chewing gum (1.32 g of xylitol), lozenges $(0.84 \mathrm{~g})$ and mouth rinses $(1 \mathrm{~g})$ [21].

Administering a chewing gum containing xylitol significantly increases the $\mathrm{pH}$ level of saliva compared to chewing gum sweetened with sucrose [29]. The report of the ADA on non-fluoride caries-preventive agents has shown that xylitol chewing gum has the best anti-carious effect [14]. The panel concluded that chewing gum should be recommended only for neurologically healthy individuals above 5 years old (due to choking haz- ard), willing to chew for a longer period [14]. The panel stated that chewing gum sweetened with polyols should be advised both for healthy children above 5 years old and adults who are at high risk of caries, though the recommendation was not unanimous [14].

Using xylitol-containing chewing gum without brushing teeth may reduce gingivitis. Bleeding from the gums during probing in individuals applying chewing gum with xylitol increased by 0.26 ; in individuals not using chewing gum - by 0.36 [30]. According to Fraga et al. [31], a high frequency (5 times a day) of applying chewing gum containing $15 \%$ of xylitol, despite the low daily total intake of xylitol ( $1 \mathrm{~g})$, depletes the amount of SM in saliva. After 30 days of discontinuation, a decrease in the count of SM in saliva was noted, compared to the first evaluation where $\mathrm{SM} \geq 10^{5} \mathrm{CFU} \mathrm{mL} /$ saliva. A study by Ly et al. [12] has proved that ingesting $10.32 \mathrm{~g}$ of xylitol in chewing gum daily results in a significant reduction of the SM count in resting saliva and dental plaque and that there is an inverse proportion between the frequency of using xylitol chewing gum and the bacteria count. Antonio et al. [17], in their literature review regarding candies and lozenges, have noted that such means of applying xylitol may decrease the increment of caries, however they do not appear effective 
toward the interproximal surfaces of teeth. Shortterm application of chewing gum reduces the SM count in saliva but does not affect the composition of oral microbiota [32]. Using chewing gum (1.33 $\mathrm{g}$ of xylitol in 1 piece 4 times per day) over 3 months is sufficient to prevent the increase of SM count in plaque [33]. Research by Runnel et al. [34] suggests that the efficacy of xylitol in caries prophylaxis may depend not only on the frequency of administration during the day but also on an even disposition of servings in time; also, a discontinuation of application, despite its latter resumption, might be significant. In conditions when retaining the usual routine of hygiene is impossible, chewing gum containing xylitol not only reduces the increment of bacteria in saliva but also improves the subjective evaluation of personal oral cavity condition [35]. According to the authors, both chewing gum and candies are recommended, considering the prolonged exposure time of the oral cavity to the xylitol contained.

According to Söderling [5], chewing gum with xylitol up to 3 times daily in pregnant females, and then new mothers, significantly reduces motherto-child SM transmission, though the relevance of sanation of the mother's oral cavity and preservation of appropriate dietary and hygienic instructions should not be neglected. Research by Thorild et al. [2] has shown that administering chewing gum with xylitol $(0.65 \mathrm{~g}$ of xylitol in 1 piece 3 times a day) by new mothers causes reduced caries occurrence, compared to using chewing gum with the addition of fluoride and sorbitol, but containing less xylitol. According to a meta-analysis of clinical trials with randomization regarding the reduction of the mother-to-child transmission of $\mathrm{SM}$, chewing gum with xylitol causes a statistically-significant depletion of SM transmission, compared to using chewing gum with sorbitol or fluoride or chlorhexidine varnish [36].

Manufacturers of chewing gum do not specify the amount that should be administered daily in order to achieve a quantifiable prophylactic effect, and, according to our calculations, while using 1 piece of chewing gum after every meal (assuming 5 meals per day) the recommended daily dose would not be attained. Titanium dioxide, affecting the color, moistness and consistency of the products, in the majority of chewing gum, present in the form of nanomolecules, may transfer from the gastrointestinal tract to other organs and accumulate there [37]. In view of the dissemination of chewing gum, abiding by the recommended time and frequency of chewing is vital so as not to instigate bruxism, occlusal parafunctions or pathological tooth attrition. In a meta-analysis by the ADA, it is stated that the benefits of chewing gum with xylitol do not outweigh its risks and, therefore, it should not be recommended to use it after every meal [14].

Xylitol content in candies varies between $83 \%$ and $96 \%$. Similarly to chewing gum, there are no explicit instructions on the amount the customer is supposed to administer. Considering the benefits of applying xylitol lozenges or hard candies, it is recommended to use them after meals in children over 5 years old [13]. Inulin, present in one of the products, is a naturally occurring probiotic, now gaining popularity. The dose of inulin sufficient to achieve a positive influence on enteric microbiota is estimated at about 5 to $8 \mathrm{~g}$ per day [38]. In some candies, there are other substances which may positively contribute to the condition of the oral cavity, such as cocoa, containing trace amounts of theobromine, which is acknowledged for its regenerating properties, for both the mind and body, and caffeine with invigorating properties. Cinnamon demonstrates antiseptic and fungicidal action. Cardamon arouses the appetite and has slight stimulating properties. Coconut oil is said to have antibacterial, antifungal and antiviral qualities. In a review from 2015, there has been only one paper regarding the efficacy of ingesting xylitol-containing candies (7.5 g per day) in children compared to candies with sorbitol over 36 months, however it was not included in the analysis due to a lack of separate results for permanent and deciduous teeth [8].

Various additions may support the candies' anticarious action or give them complementary properties. Products with xylitol often also contain different sweeteners, such as stevia - an herbal sweetener. Studies have shown that stevia extract does not aid the development of SM and is not fermented by the components of biofilm [39]. Parsley seed oil has antibacterial, anti-inflammatory, and shrinking properties and improves blood circulation. Bromelain, a blend of protease enzymes occurring in pineapples, is believed to be anti-inflammatory and aids healing. One of the toothpastes in the present study contained eugenol, known for its antiseptic and anesthetic action. Another ingredient of the same toothpaste was cherry blossom extract, which has confirmed anti-inflammatory and soothing properties in products meant for skin care [40]. In traditional medicine, nigella is used to treat inflammation. Licorice is acknowledged for its anti-inflammatory, antiviral, antibacterial and antioxidant action; it also neutralizes redness and swelling. Many species of sage have application in medicine, and aloe aids healing and soothes irritation. However, there are still questions about the amount of certain substances used in those products and their impact on the condition of the oral cavity with reference to xylitol. 
According to the most recent Cochrane literature review, there is shortage of EBM data indicating that many of the food and dental care products containing xylitol are efficient [8]. There are a few studies of low quality suggesting that fluoride toothpastes containing xylitol may be more efficient in caries prevention in children than fluoride-only toothpastes [8]. Riley et al. [8] bring up the fact that two studies were conducted by the same authors in the same population. Other studies were evaluated as being of very low quality and were insufficient to determine whether the products containing xylitol may prevent caries in infants, children or adults.

\section{Conclusions}

Many dental care and food products available on the Polish market, recommended in caries prevention, contain xylitol in diverse concentrations. Most of the manufacturers do not give direct instructions on the amount that should be administered and do not emphasize the significance of regular xylitol ingestion. The conclusion might be drawn that replacing some of the cariogenic products with their equivalents containing xylitol is possible in many cases, and the aim should be to increase the awareness of patients, particularly parents and guardians in order to administer such products to children. If cariogenic sugars are replaced with non-cariogenic sugar polyols, the occurrence of caries will be diminished.

Though xylitol is included in many dental prophylactic programs as an acknowledged anticarious agent, used regularly and in the right concentration, there is a shortage of EBM data indicating that many of the food and dental care products containing xylitol are effective, apart from fluoride toothpastes with the addition of xylitol. According to the Cochrane literature review, there are a few low-quality studies suggesting that fluoride toothpastes with xylitol may be more efficacious in caries prevention in children than toothpastes containing only fluoride. Other studies were evaluated as of very low quality and are insufficient to determine whether products containing xylitol may prevent caries in infants, children or adults. It is indicated that more precise studies are necessary.

\section{References}

[1] Council of Clinical Affairs, Guideline on xylitol use in caries prevention. American Academy of Pediatric Dentistry. 2014/2015, Reference Manual 36, 6.

[2] Thorild I., Lindau B., Twetman S.: Caries in 4-year-old children after maternal chewing of gums containing combinations of xylitol, sorbitol, chlorhexidine and fluoride. Eur. Arch. Paediatr. Dent. 2006, 7, 241-245.

[3] Miyasawa H., Inami Y., Mayanagi H., Takahashi N.: Xylitol inhibition of anaerobic acid production by streptococcus mutans at various pH levels. Oral Microbiol. Immunol. 2003, 18, 215-219.

[4] Shinga-Ishihara C., Nakai Y., Milgrom P., Söderling E., Tolvanen M., Murakami K.: Xylitol carryover effects on salivary mutans streptococci after 13 months of chewing xylitol gum. Caries Res. 2012, 46, 519-522.

[5] SöDerling E.M.: Xylitol, mutans streptococci, and dental plaque. Adv. Dent. Res. 2009, 21, 74-78.

[6] Mäkinen K.K., SaAg M., Isotupa K.P., Olak J., Nõmmela R., Söderling E., MäKinen P.L.: Similarity of the effects of erythritol and xylitol on some risk factors of dental caries. Caries Res. 2005, 39, 207-215.

[7] Tanzer J.M., Thompson A., Wen Z.T., Burne R.A.: Streptococcus mutans: Fructose transport, xylitol resistance, and virulence. J. Dent. Res. 2006, 85, 369-373.

[8] Riley P., Moore D., Ahmed F., Sharif M.O., Worthington H.V.: Xylitol-containing products for preventing dental caries in children and adults. Cochrane Database Syst. Rev. 2015, 26, 3, CD010743.

[9] Campus G., Cagetti M.G., Sale S., Petruzzi M., Solinas G., Strohmenger L., Lingström P.: Six months of high-dose xylitol in high-risk caries subjects - a 2-year randomised, clinical trial. Clin. Oral Investig. 2013, 17, 785-791.

[10] Ritter A.V., Bader J.D., Leo M.C., Preisser J.S., Shugars D.A., Vollmer W.M., Amaechi B.T., Holland J.C.: Tooth-surface-specific effects of xylitol: Randomized trial results. J. Dent. Res. 2013, 92, 512-517.

[11] Milgrom P., Ly K.A., Roberts M.C., Rothen M., Mueller G., Yamaguchi D.K.: Mutans streptococci dose response to xylitol chewing gum. J. Dent. Res. 2006, 85, 177-181.

[12] Ly K.A., Milgrom P., Roberts M.C., Yamaguchi D.K., Rothen M., Mueller G.: Linear response of mutans streptococci to increasing frequency of xylitol chewing gum use: A randomized controlled trial. BMC Oral Health 2006, 24, 6 .

[13] Oscarson P., Lif Holgerson P., Sjöström I., Twetman S., Stecksén-Blicks C.: Influence of a low xylitol-dose on mutans streptococci colonisation and caries development in preschool children. Eur. Arch. Paediatr. Dent. 2006, 7, 142-147.

[14] ADA Center for Evidence Based Dentistry: Non-fluoride caries preventive agents, full report of a systematic review and evidence-based recommendations. A report of the council on scientific affairs 2011, 24, 5.

[15] Lingström P., Holm A.K., Mejàre I., Twetman S., Söder B., Norlund A., Axelsson S., Lagerlöf F., Nordenram G., Petersson L.G., Dahlgren H., Källestål C.: Dietary factors in the prevention of dental caries: A systematic review. Acta Odontol Scand. 2003, 61, 331-340.

[16] Fontana M., González-Cabezas C.: Are we ready for definitive clinical guidelines on xylitol/polyol use? Adv. Dent. Res. 2012, 24, 123-128. 
[17] Antonio A.G., Pierro V.S., Maia L.C.: Caries preventive effects of xylitol-based candies and lozenges: A systematic review. J. Public Health Dent. 2011, 71, 117-124.

[18] Chi D.L., Tut O., Milgrom P.: Cluster-randomized xylitol toothpaste trial for early childhood caries prevention. J. Dent. Child. (Chic.). 2014, 81, 27-32.

[19] Sano H., Nakashima S., Songpaisan Y., Phantumvanit P.: Effect of a xylitol and fluoride containing toothpaste on the remineralization of human enamel in vitro. J. Oral Sci. 2007, 49, 67-73.

[20] ElSalhy M., Sayed Zahid I., Honkala E.: Effects of xylitol mouthrinse on Streptococcus mutans. J. Dent. 2012, $40,1151-1154$.

[21] Lif Holgerson P., Stecksén-Blicks C., Sjöström I., Oberg M., Twetman S.: Xylitol concentration in saliva and dental plaque after use of various xylitol-containing products. Caries Res. 2006, 40, 393-397.

[22] Hildebrandt G., Lee I., Hodges J.: Oral mutans streptococci levels following use of a xylitol mouth rinse: A double-blind, randomized, controlled clinical trial. Spec. Care Dent. 2010, 30, 53-58.

[23] Subramaniam P., Nandan N.: Effect of xylitol, sodium fluoride and triclosan containing mouth rinse on streptococcus mutans. Contemp. Clin. Dent. 2011, 4, 287-290.

[24] Decker E.M., Maier G., Axmann D., Brecx M., von Ohle C.: Effect of xylitol/chlorhexidine versus xylitol or chlorhexidine as single rinses on initial biofilm formation of cariogenic streptococci. Quintessence Int. 2008, 39, 17-22.

[25] Zhan L., Cheng J., Chang P., Ngo M., DenBesten P.K., Hoover C.I., Featherstone J.D.B.: Effects of xylitol wipes on cariogenic bacteria and caries in young children. J. Dent. Res. 2012, 91, 85-90.

[26] Mäkinen K.K., Järvinen K.L., Anttila C.H., Luntamo L.M., Vahlberg T.: Topical xylitol administration by parents for the promotion of oral health in infants: A caries prevention experiment at a Finnish Public Health Centre. Int. Dent. J. 2013, 63, 210-224.

[27] Milgrom P., Ly K.A., Tut O.K., Mancl L., Roberts M.C., Briand K., Gancio M.J.: Xylitol pediatric topical oral syrup to prevent dental caries: A double-blind randomized clinical trial of efficacy. Arch. Pediatr. Adolesc. Med. 2009, 163, 601-607.

[28] Assev S., STig S., SCheie A.A.: Cariogenic traits in xylitol-resistant and xylitol-sensitive mutans streptococci. Oral Microbiol. Immunol. 2002, 17, 95-99.

[29] Kumar S., Sogi S.H., Indushekar K.R.: Comparative evaluation of the effects of xylitol and sugar-free chewing gums on salivary and dental plaque pH in children. J. Indian Soc. Pedod. Prev. Dent. 2013, 31, 240-244.

[30] Keukenmeester R.S., Slot D.E., Rosema N.A., Van Loveren C., Van der Weijden G.A.: Effects of sugar-free chewing gum sweetened with xylitol or maltitol on the development of gingivitis and plaque: A randomized clinical trial. Int. J. Dent. Hyg. 2014, 12, 238-244.

[31] Fraga C.P., Mayer M.P., Rodrigues C.R.: Use of chewing gum containing 15\% of xylitol and reduction in $m u$ tans streptococci salivary levels. Braz. Oral Res. 2010, 24, 142-146.

[32] Söderling E., ElSalhy M., Honkala E., Fontana M., Flannagan S., Eckert G., Kokaras A., Paster B., Tolvanen M., Honkala S.: Effects of short-term xylitol gum chewing on the oral microbiome. Clin. Oral Investig. 2015, 19, 237-244.

[33] Seki M., Karakama F., Kawato T., Tanaka H., Saeki Y., Yamashita Y.: Effect of xylitol gum on the level of oral mutans streptococci of preschoolers: Block-randomized trial. Int. Dent. J. 2011, 61, 274-280.

[34] Runnel R., Mäkinen K.K., Honkala S., Olak J., Mäkinen P.L., Nõmmela R., Vahlberg T., Honkala E., SAAG M.: Effect of three-year consumption of erythritol, xylitol and sorbitol candies on various plaque and salivary caries-related variables. J. Dent. 2013, 41, 1236-1244.

[35] Hashiba T., Takeuchi K., Shimazaki Y., Takeshita T., Yamashita Y.: Chewing xylitol gum improves self-rated and objective indicators of oral health status under conditions interrupting regular oral hygiene. Tohoku J. Exp. Med. 2015, 235, 39-46.

[36] Lin H.K., Fang C.E., Huang M.S., Cheng H.C., Huang T.W., Chang H.T., Tam K.W.: Effect of maternal use of chewing gums containing xylitol on transmission of mutans streptococci in children: A meta-analysis of randomized controlled trials. Int. J. Paediatr. Dent. 2016, 26, 35-44.

[37] Chen X.X., Cheng B., Yang Y.X., Cao A., Liu J.H., Du L.J., Liv Y., Zhao Y., Wang H.: Characterization and preliminary toxicity assay of nano-titanium dioxide additive in sugar-coated chewing gum. Small, 2013, 9, 1765-1774.

[38] Kolida S., Gibson G.R.: Prebiotic capacity of inulin-type fructans. J. Nutr. 2007, 137, 2503-2506.

[39] Brambilla E., Cagetti M.G., Ionescu A., Campus G., Lingström P.: An in vitro and in vivo comparison of the effect of Stevia rebaudiana extracts on different caries-related variables: A randomized controlled trial pilot study. Caries Res. 2014, 48, 19-23.

[40] Zhang Y.Q., Guan L., Zhong Z.Y., Chang M., Zhang D.K., Li H., LaI W.: The anti-inflammatory effect of cherry blossom extract (Prunus yedoensis) used in soothing skincare product. Int. J. Cosmet. Sci. 2014, 36, 527-530.

\section{Address for correspondence:}

Anna Turska-Szybka

Department of Paedodontics

Medical University of Warsaw

Miodowa 18

00-246 Warsaw

Poland

Tel.: 225022031

E-mail: aturskaszybka@orange.pl

Conflict of Interest: None declared

Received: 17.04.2016

Revised: 27.05.2016

Accepted: 28.06.2016 